\title{
Microwave-assisted Fixation, Labeling and Clearing for Optical Microscopy of Thick Specimens
}

\author{
Adam Vogel, Guillermo Marqués, and Mark A. Sanders \\ University Imaging Centers, University of Minnesota, St. Paul, MN, USA 55108
}

Recent innovations in microscope design have increased working distances of optics permitting the collection of subcelluar fluorescence details at depths greater than several millimeters. Sample preparation methodologies have evolved to take advantage of these capabilities, however these protocols take days to months to prepare the tissue for imaging (1). Intact tissue microscopy typically involves fixing and staining, followed by clearing and mounting. Clearing involves treatment with an agent to obviate components that cause light scattering and reduce the ability to image through thick specimens while maintaining morphological and molecular profiles. A wide variety of clearing and mounting media for microscopy have been described in the literature; in several cases the same media are used for both clearing and mounting. Careful attention to clearing and mounting has enabled confocal microscopy of samples greater than $4 \mathrm{~mm}$ thick (2). A drawback is that this method requires multiple weeks of clearing time.

Parallel innovation in microwave-assisted processing has been demonstrated to contribute toward accelerating a wide range of processing applications; including live cell imaging, fixations, labeling and embedding, all while preserving probe intensity (3). In this study we have attempted to identify optimal sample preparation, clearing and mounting steps for deep tissue microscopy while significantly shortening the processing time. To this end we tested five commonly used mounting and clearing reagents either hydrophilic or hydrophobic in nature (Table 1). In all cases, we used the same media for clearing and mounting. Porcine skeletal muscle was utilized in this study as a good test of the ability to image deep into a dense, yet uniform, specimen.

Fresh porcine muscle tissue was grossed to roughly $5 \mathrm{~mm}$ thick slices and uniform $5 \mathrm{~mm}$ punches of tissue were collected. Experimental and control tissue was prepared as in Table 2 utilizing a Ted Pella BioWave Pro microwave using a ColdSpot ${ }^{\mathrm{TM}}$ set to $4^{\circ} \mathrm{C}$ and vacuum chamber. The sample tempertures were monitored during processing and did not exceed $25^{\circ} \mathrm{C}$. The tissue was labeled with $2.0 \mu \mathrm{M}$ of the far-red excited fluorescent DNA stain To-Pro3 (Life Technologies) to observe nuclei. Once processed, samples were mounted in their respective clearing agent and sealed with No. 1.5 coverslips.

Replicate samples were imaged using a Nikon A1R MP confocal in resonance mode with an Apo LWD 25x/1.1 NA immersion lens and a Nikon A1 confocal microscope using a Plan Fluor 20x/0.75 NA objective. Z-stacks were acquired at appropriate Nyquest sampling steps through 400 microns of the tissue resulting in a voxel size of 160x160x375 nm and 250x250x $875 \mathrm{~nm}$ respectively. 3D volumes of these stacks are shown in Figure 1. A depth of approximately $350 \mu \mathrm{m}$ was reached with microwaveassisted glycerol treatment when observed using the higher NA immersion objective as shown in Figure 2. Significant photobleaching was not observed.

Our findings suggest that microwave-assisted processing increases functional imaging depths on average of $40 \%$ greater than parallel treatment samples in the absence of microwaves at identical processing times. Glycerol appears to be an optimal mounting medium when imaging depth is important in an air objective and Spalteholz using an immersion objective. Microwave-assisted processing demonstrates an overall 10-100 fold time-savings while giving improved sample preservation at depth in these 
treatments. We will report on the comparisons of microwave-assisted clearing treatments for optimal clearing for both air and immersion optics.

\section{References:}

1. http://nic.ucsf.edu/dokuwiki/lib/exe/fetch.php?media=mounting_media_tests.pdf

2. Hama H, Kurokawa H, Kawano H, Ando R, Shimogori T, et al. (2011) Scale: a chemical approach for fluorescence imaging and reconstruction of transparent mouse brain. Nat Neurosci 14(11): 1481-1490.

3. Ferris AM, Giberson RT, Sanders MA, Day JR. (2009) Advanced laboratory techniques for sample processing and immunolabeling using microwave radiation. Journal of Neuroscience Methods. 182(2): 157-164.

4. Spalteholz W (1914) Über das Durchsichtigmachen von menschlichen und tierischen Präparaten. S. Hierzel, Leipzig.

Table 1. Clearing Reagents

\begin{tabular}{|l|l|c|l|}
\hline \multicolumn{1}{|c|}{ Name } & \multicolumn{1}{c|}{ Composition } & R.I. & \multicolumn{1}{c|}{ Notes } \\
\hline \hline BABB & 33\% Benzyl Alcohol: 67\% Benzyl Benzoate & 1.56 & Requires dehydration \\
\hline *Glycerol & Glycerol & 1.47 & Dehydration not required \\
\hline Methyl Salicylate & $100 \%$ Methyl Salicylate & 1.536 & Requires dehydration \\
\hline *Spalteholz (4) & $5: 3$ Methyl Salicylate:Benzyl Benzoate & 1.518 & Requires dehydration \\
\hline TDE & $97 \% 2,2$ '-Thiodiethanol in water & 1.515 & Dehydration not required \\
\hline
\end{tabular}

Table 2. Microwave-assisted protocol

\begin{tabular}{|c|c|c|c|c|}
\hline Step & Description & Time (on:off:on) min & Power (Watts) & Vacuum (Hg) \\
\hline 1 & Fix tissue in $4 \%$ paraformaldehyde (in PBS $\mathrm{pH} 7.2$ ) & 10 & 150 & Off \\
\hline 2 & Continue fixing tissue in $4 \%$ paraformaldehyde & 5 & 250 & Off \\
\hline 3 & $3 \mathrm{x}$ PBS-T $(0.1 \%$ Triton $\mathrm{x}-100)$ rinse & 2 & 150 & Off \\
\hline 4 & $0.2 \mu \mathrm{M}$ ToPro-3 (in PBS pH 7.2) & 6:03:06 & 150 & Off \\
\hline 5 & $3 x$ PBS-T rinse & 2 & 150 & Off \\
\hline 6 & $2 \times 75 \% \mathrm{EtOH}$ & 5 & 150 & 20 \\
\hline 7 & $2 \times 85 \%$ EtOH & 5 & 150 & 20 \\
\hline 7 & $2 \times 95 \% \mathrm{EtOH}$ & 5:02:05 & 150 & 20 \\
\hline 8 & $3 \times 100 \% \mathrm{EtOH}$ & 5 & 150 & 20 \\
\hline \multirow[t]{2}{*}{9} & $5 \mathrm{x}$ Clearing solution (repeat until cleared for larger tissues) & 6:03:06 & 150 & 20 \\
\hline & TOTAL TIME & $<180 \mathrm{~min}$ & & \\
\hline
\end{tabular}
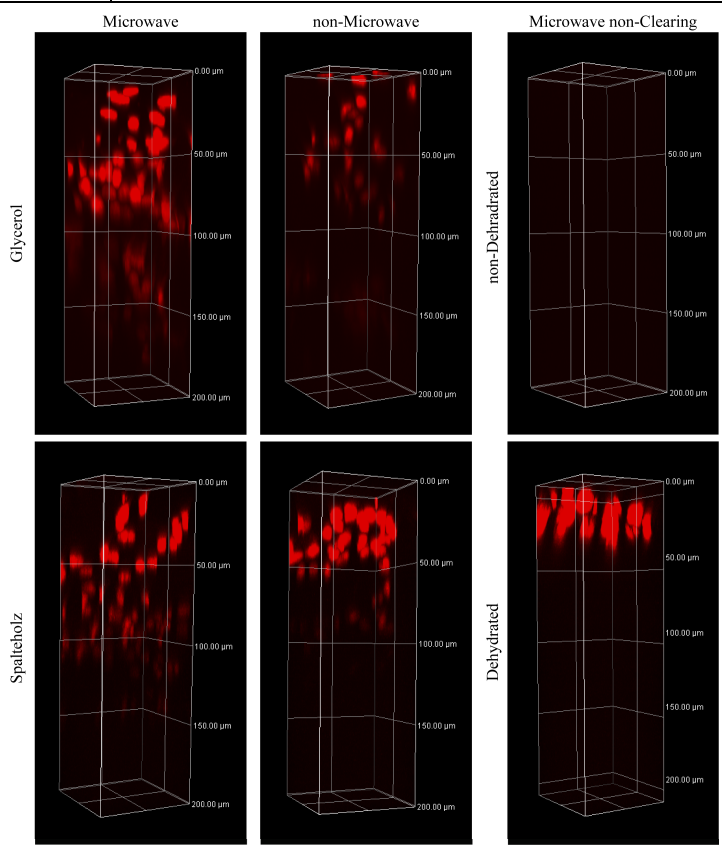

Figure 1: 3D projection of porcine muscle acquired with a NikonA1comparing MW, non-MW and control treatments. $2 \mu \mathrm{M}$ To-Pro3.

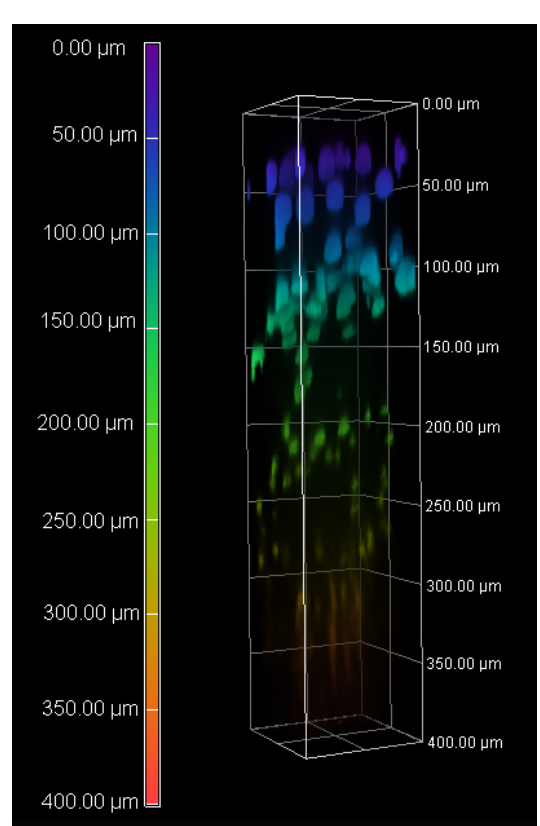

Figure 2: Glycerol MW sampling showing full $400 \mu \mathrm{m}$ using a Nikon $25 \mathrm{x}, 1.1 \mathrm{NA}$ immersion objective. Colormap corresponds to signal depth of To-Pro3 labeling. 